\title{
The Efficacy of Argon Plasma Coagulation versus Carvedilol for Treatment of Portal Hypertensive Gastropathy
}

\author{
Mohamed S. El Shahawy ${ }^{a}$ Zakarya M. Shady a Abdullah Gaafar ${ }^{b}$ \\ a Department of Internal Medicine, Gastroenterology Unit, Faculty of Medicine, AL-Azhar University, Cairo, Egypt; \\ ${ }^{b}$ Department of Clinical Pathology, Faculty of Medicine, AL-Azhar University, Cairo, Egypt
}

\section{Keywords}

Portal hypertensive gastropathy · Argon plasma

coagulation · Carvedilol

\begin{abstract}
Background/Aim: Several treatment options have been developed for portal hypertensive gastropathy (PHG); medications and endoscopic management. The aim of this study was to evaluate the efficacy and safety of argon plasma coagulation (APC) versus Carvedilol in treatment of a cohort of Egyptian patients with severe PHG. Methods: A total of 130 patients with severe PHG were enrolled; 10 patients were excluded due to death and failure to complete the treatment sessions accordingly, 120 patients were included. Patients were divided into 2 groups: Group A $(n=52)$ treated with APC; Group B $(n=68)$ treated with oral Carvedilol. Success was defined as stabilization of hemoglobin $(\mathrm{Hb})$ over $100 \mathrm{~g} /$ $\mathrm{dL}$ or $\mathrm{Hb}$ increase $>10 \%$ from pretreatment level and reduction of blood transfusion requirements over the following 3 months after the start of therapy. Upper gastrointestinal endoscopy was performed to assess the degree and site of PHG. APC was conducted to areas with mucosal ectatic vascular lesions. Results: PHG was mostly fundic (36.35\%) in APC group and (36.76\%) in Carvedilol group ( $p=0.56$ ). Throughout follow-up period, there was significant increase
\end{abstract}

in $\mathrm{Hb}$ level, serum iron, and serum ferritin with a significant decrease in total iron-binding capacity (TIBC) in APC group as compared to Carvedilol group ( $p<0.001)$. Additionally, there was gradual increase in the mean $\mathrm{Hb}$, serum iron, and serum ferritin and gradual decrease of TIBC in Carvedilol group. Accordingly, there was an overall improvement of iron deficiency anemia (IDA) in both groups; however, it was significantly better in APC group than in Carvedilol group. No major adverse events were detected in both the groups. Conclusion: APC significantly improves IDA and decreases transfusion requirements in patients with severe PHG as compared to oral Carvedilol with small risk of adverse events. Furthermore, the combination of APC and Carvedilol unless contraindicated could have a synergistic effect in controlling severe PHG.

○) 2019 S. Karger AG, Basel

\section{Introduction}

Portal hypertension $(\mathrm{PH})$ is an almost unavoidable complication of cirrhosis and provides the driving force for most of its complications, such as esophageal and gastric varices, variceal bleeding, portal hypertensive gastropathy (PHG), ascites, spontaneous bacterial peritonitis, hepatorenal syndrome, thrombopenia, leucopenia,

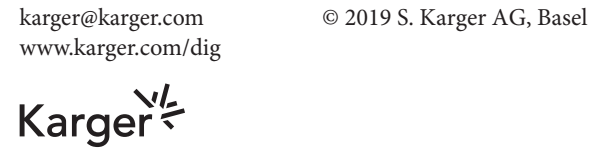

Mohamed S. El Shahawy 
anemia, and portal-systemic encephalopathy [1]. Because of this, there is a great interest in strategies to revert $\mathrm{PH}$, since these would prevent $\mathrm{PH}$-related complications, clinical decompensation, and death. PHG accounts for $8 \%$ of nonvariceal upper gastrointestinal (GI) bleeding in patients with liver disease [2]. The gold standard method of determining portal pressure involves accessing the hepatic vein through a femoral catheter and measuring the wedged and free pressures. The difference of these 2 pressures is called the hepatic venous pressure gradient (HVPG), which is closely related to the development of complications of $\mathrm{PH}$. The elevation of HVPG from a normal of $1-3 \mathrm{~mm} \mathrm{Hg}$ to $>12$ is associated with development of ascites and greater chances of variceal rupture. Severe $\mathrm{PH}$ is usually defined as an HVPG $>15 \mathrm{~mm} \mathrm{Hg}$ [3]. PHG is classified endoscopically according to McCormack et al. [4] who first described it in 1985; however, it was limited by lack of grading of intermediate endoscopic findings. In 1994, the New Italian Endoscopy Club (NIEC) proposed an alternative classification including a moderate aspect of PHG [5]. Several treatment options have been developed for PHG; medications and endoscopic management. Argon plasma coagulation (APC) has been used to stop bleeding from ectatic vessels, and to attempt to obliterate the vessels, but have limited utility if the disease is diffuse [6]. APC is an electrosurgical technique for the management of bleeding and the devitalization of tissue abnormalities. This is achieved by a noncontact thermal coagulation in which high-frequency current is applied to the target tissue through an argon plasma jet creating effective hemostasis and a homogenous surface coagulation with a limited penetration depth. It was reported that the hemoglobin $(\mathrm{Hb})$ value improved and transfusion requirements decreased in patients with PHG after therapy with APC [7]. Conventionally, the nonselective $\alpha$-/ $\beta$-blocker, propranolol has been used to lower portal pressure and to avoid variceal rupture and consequent hematemesis. However, two-thirds of cirrhotic patients may not respond to propranolol [8]. Carvedilol is a newer nonselective $\beta$-antagonist that also possesses $\alpha 1$ antagonist receptor properties is currently a mainstay in the treatment of heart failure and hypertension. It is $2-4$ times powerful as compared to propranolol in its $\beta$-blocking properties [9]. Carvedilol is a potent nonselective beta-blocker with mild anti-alpha 1 adrenergic activity (one-tenth of its beta-blocker activity). It was developed for the treatment of arterial hypertension and heart failure and has US. Food and Drug Administration approval for both indications. Milligram for milligram, Carvedilol is 2-4 times more potent than propranolol as a beta-receptor antagonist [10]. Carvedilol decreases heart rate and cardiac output and causes splanchnic vasoconstriction. This results in reduced portal blood inflow and in a fall in portal pressure. In addition, through its alpha 1 adrenoceptor blocking effect, Carvedilol decreases the hepatic vascular tone and hepatic resistance resulting in a further decrease in portal pressure [1]. However, the vasodilating activity of Carvedilol may enhance arterial hypotension and sodium retention, a risk which is especially relevant in patients with advanced, decompensated cirrhosis [11]. Some studies suggest that relatively low doses of Carvedilol (12.5 mg/day or $6.25 \mathrm{mg}$ bid) retain a good portal pressure reducing effect with less potential for hypotension which may be relevant when treating decompensated patients with cirrhosis [12].

\section{Materials and Methods}

This prospective, observational study was conducted at ELHussein university hospital, AL-Azhar University, Cairo, Egypt, between March 2018 and May 2019. This study was approved by Institutional Review Board of the Faculty of Medicine (AL-Azhar Institutional Ethics Committee), and all study subjects provided informed consent.

\section{Study Design}

Adult patient's $\geq 18$ years old were eligible for recruitment if they had hepatic disease and with severe PHG diagnosed by upper GI endoscopy. The indications of upper GI endoscopy were recurrent episode of upper GI bleeding or during the follow-up after variceal endoscopic therapy and chronic iron deficiency anemia (IDA) resistant to conventional therapy. Patients were excluded if they were in an acute episode of hepatic encephalopathy, severe cardiopulmonary disease, chronic renal failure or hemodynamic instability, or if they had actively bleeding esophageal or gastric varices, or actively bleeding gastric or duodenal ulcers or malignancies and finally, patients with known allergy or contraindication to Carvedilol (bronchial asthma, decompensated heart failure, etc.). Patients were divided into 2 groups: APC Group $(n=52)$ treated with APC; Group B $(n=68)$ treated with oral Carvedilol. The selected groups of patients were subjected to the following: (1) full history taking, (2) complete clinical examination: vital signs, signs of $\mathrm{PH}$ such as dilated abdominal veins, or splenomegaly; signs of liver cell failure such as jaundice, ascites, lower limb edema, fetor hepaticus, flapping tremors, or spider angioma; and signs of renal, cardiac, or respiratory diseases; (3) laboratory investigations: liver function tests, prothrombin time, prothrombin concentration (\%), and INR; kidney function tests including blood urea and serum creatinine; complete blood count; and iron profile. For each patient, the Child-Pugh score was calculated. Abdominal ultrasonography for all patients was conducted, and cirrhotic echo pattern was determined from the coarse nodular appearance and shrunken size with prominent caudate lobe. The following were also noted: presence of ascites, portal vein diameter, splenic bipolar diameter, hilar varices, and splenic vein diameter. 


\section{Endoscopic Evaluation}

The instruments used in this study were a videoendoscope and an electronic endoscopic system (Olympus EVIS Lucera Ellit CV290; Olympus Medical Systems, Tokyo, Japan) equipped with 3 imaging modes: high-resolution white light endoscopy, autofluorescence imaging, and narrow-band imaging. Mode switch from white light endoscopy to narrow-band imaging or autofluorescence imaging was controlled by buttons on the control head. All patients were offered conscious sedation with intravenous midazolam (2.5-7.5 mg) and/or propofol (40-200 mg) and then underwent endoscopic assessment of the degree and site of PHG, presence of esophageal varices and their grade, fundal extension or presence of isolated fundal varix, gastritis or duodenitis, erosions, and ulcerations. PHG was assessed according to the NIEC classifications. The elementary lesions of PHG according to the NIEC classification are as follows: (1) mosaic-like pattern is defined as the presence of small, polygonal areas surrounded by a whitish-yellow depressed border. The mosaic is defined as mild when the areola is uniformly pink, moderate if the center is red, and severe if the areola is uniformly red. (2) Red-point lesions (RPLs) are small, flat, red point-like lesions, $1 \mathrm{~mm}$ in diameter. (3) Cherry-red spots (CRSs) are red-colored, round lesions about 2 $\mathrm{mm}$ in diameter, and slightly protrude into the lumen of the stomach. (4) Black-brown spots (BBSs) are irregularly shaped flat spots, black or brown, persistently present after washing, and caused by intramucosal hemorrhage. At a previous International Consensus Conference, it was decided to define PHG as mild when only mosaic-like pattern of any degree was present and severe when RPLs, CRSs, or BBSs were present [5]. Esophageal varices was classified according to Westaby classification of esophageal varices [13], which has been endorsed by the British Society of Gastroenterology in its guidelines [14], Grade 1: Varices appearing as slight protrusion above mucosa, which can be depressed with insufflations; Grade 2: Varices occupying $<50 \%$ of the lumen; Grade 3: Varices occupying $>50 \%$ of the lumen and which are very close to each other with confluent appearance. All of the endoscopic examinations were performed by one senior endoscopes with $>10$ years of experience in diagnostic and therapeutic endoscopy.

\section{Argon Plasma Coagulation}

The APC system was consisted of the following: the disposable APC probe with diameters of $1.5 \mathrm{~mm}, 2.3 \mathrm{~mm}$, and $220 \mathrm{~cm}$ long, a foot pedal, 2 tanks of argon gas, and a high-frequency monopole electrosurgical generator current conducted to target tissues through ionized argon gas (argon plasma). Electrical spark could be easily elicited by pressing the blue foot pad connected to APC equipment. The argon gas flow was set at $2.5 \mathrm{~L} / \mathrm{min}$, and the electrical power output was adjusted to $60-90 \mathrm{~W}$. APC was applied to all areas of visible red spots of congested gastric mucosa with a depth of penetration of roughly $2-3 \mathrm{~mm}$ for about $1-3 \mathrm{~s}$. The session duration was from 15 to $20 \mathrm{~min}$. APC was done in a single session in patients who have localized PHG and in multiple sessions in patients who have diffuse PHG where endoscopy was repeated after 2 weeks. All patients received intravenous antibiotics therapy after the procedure (ceftriaxone I $g$ daily) for prophylaxis of infection as well, proton pump inhibitor therapy (lansoprazole $30 \mathrm{mg}$ daily) therapy after the procedure to improve mucosal healing.

APC versus Carvedilol for PHG

\section{Carvedilol Dose}

Carvedilol doses were administrated at 12.5-25 mg (mean $19 \pm$ $3.79 \mathrm{mg} /$ day) starting at $6.25 \mathrm{mg}$ and titrated up every 4 days according to blood pressure and heart rate intended to reduce the heart rate by $25 \%$ or down to 55 beats $/ \mathrm{min}$. The dose should not be increased in patients developing symptoms or with a systolic blood pressure $<90 \mathrm{~mm} \mathrm{Hg}$ or a heart rate $<50 \mathrm{bpm}$. Carvedilol is contraindicated in patients with marked bradycardia, the sick sinus syndrome, and partial or complete heart block (unless a pacemaker is in place). Thus, an electrocardiogram is mandatory before starting therapy. Carvedilol is also contraindicated in patients with asthma. Carvedilol was used cautiously in patients with insulin-dependent diabetes because it can mask the symptoms of hypoglycemia.

\section{Outcome Measures}

All patients were followed up after treatment by laboratory evaluation of $\mathrm{Hb}$ level and iron profile. Success was defined as; stabilization of $\mathrm{Hb}$ over $100 \mathrm{~g} / \mathrm{dL}$, or $\mathrm{Hb}$ increase $>10 \%$ from pretreatment level, and reduction of transfusion requirements $>50 \%$ in transfusion-dependent patients over the following 3 months after the start of therapy.

\section{Statistical Analysis}

Data were analyzed by statistical package for social science (version 16.0; SPSS Inc., Chicago, IL, USA). Quantitative data were expressed as mean $\pm \mathrm{SD}$. General linear model was used to test differences at different times using repeated measures analysis of variance. Paired $t$ test was used to test before and after paired observations. Qualitative data were expressed as numbers and percentages and analyzed by chi-square test. For the calculation of cutoff values, the receiver operator curve was calculated and the best cutoff was determined. For correlation between 2 paramours, the Pearson's correlation coefficient $(r)$ was calculated; it was inversed if the sign is negative and proportional if the sign is positive. Level of significance was set to a $p$ value $<0.05$.

\section{Results}

A total of 130 patients were recruited over the study period; 10 patients $(8.33 \%)$ were excluded due to either death $(n=5)$ caused by hepatic encephalopathy and hepatorenal syndrome or did not complete the treatment sessions $(n=$ 5); therefore, 120 patients completed the study (92.30\%). Table 1 presents the baseline demographic and clinical characteristics for the 2 groups. Of the 120 patients, 90 (75\%) were referred to the emergency endoscopy unit due to either melena or hematemesis and $30(25 \%)$ were referred for elective upper GI endoscopy as a follow-up after variceal endoscopic therapy. The mean $\mathrm{Hb}$ level at the start of therapy of both APC and Carvedilol groups was $7.95 \pm$ $0.52,7.42 \pm 0.33$, respectively, without significant difference $(p=0.17)$. The most common cause of PHG was viral hepatitis 57 (47.5\%) including HCV 44 (36.6\%) and HBV $13(10.8 \%)$ followed by viral hepatitis mixed with bilharziasis $51(42.5 \%)$ and finally bilharziasis alone $12(10 \%)$. 
Table 1. Baseline characteristics of the study groups

\begin{tabular}{|c|c|c|c|}
\hline Variable & $\begin{array}{l}\text { APC group } \\
(n=52)\end{array}$ & $\begin{array}{l}\text { Carvedilol group } \\
(n=68)\end{array}$ & $p$ value \\
\hline Age, years, mean \pm SD & $45.9 \pm 8.6$ & $46.5 \pm 7.6$ & 0.18 \\
\hline \multicolumn{4}{|l|}{ Gender, $n(\%)$} \\
\hline Male & $33(63.46)$ & $42(61.76)$ & 0.67 \\
\hline Female & $19(36.53)$ & $26(38.23)$ & 0.68 \\
\hline ALT, IU/L & $44.05 \pm 10.34$ & $46.04 \pm 8.7$ & 0.87 \\
\hline $\mathrm{AST}, \mathrm{IU} / \mathrm{L}$ & $37.92 \pm 12.52$ & $39.76 \pm 11.54$ & 0.95 \\
\hline $\mathrm{INR}$, mean $\pm \mathrm{SD}$ & $2.05 \pm 0.44$ & $1.06 \pm 0.32$ & 0.92 \\
\hline Bilirubin (total), mg/dL & $3.30 \pm 1.53$ & $3.29 \pm 1.64$ & 0.45 \\
\hline Albumin, mean $\pm \mathrm{SD}, \mathrm{g} / \mathrm{dL}$ & $2.70 \pm 0.70$ & $2.60 \pm 0.65$ & 0.89 \\
\hline Urea, mean $\pm \mathrm{SD}, \mathrm{mg} / \mathrm{dL}$ & $65 \pm 15$ & $66 \pm 14$ & 0.78 \\
\hline Creatinine, mean $\pm \mathrm{SD}, \mathrm{mg} / \mathrm{dL}$ & $1.23 \pm 0.3$ & $1.21 \pm 0.4$ & 0.96 \\
\hline $\mathrm{Hb}, \mathrm{g} / \mathrm{dL}$ & $7.95 \pm 0.52$ & $7.42 \pm 0.33$ & 0.17 \\
\hline WBCs & $4.69 \pm 3.87$ & $3.9 \pm 1.95$ & 0.28 \\
\hline Platelets & $89.27 \pm 45.87$ & $90.8 \pm 25.11$ & 0.67 \\
\hline Iron, $\mu \mathrm{g} / \mathrm{dL}$ & $51.37 \pm 2.72$ & $50.40 \pm 3.75$ & 0.88 \\
\hline TIBC, $\mu \mathrm{g} / \mathrm{dL}$ & $476.64 \pm 19.34$ & $478.28 \pm 20.04$ & 0.62 \\
\hline Ferritin, $\mu \mathrm{g} / \mathrm{dL}$ & $9.12 \pm 0.45$ & $8.96 \pm 0.57$ & 0.29 \\
\hline Ascites, $n(\%)$ & $43(82.69)$ & $56(82.35)$ & 0.58 \\
\hline Spleen, $n(\%)$ & & & 0.67 \\
\hline Normal & $3(5.35)$ & $4(5.88)$ & \\
\hline Splenectomy & $4(7.14)$ & $5(7.35)$ & \\
\hline Splenomegally & $45(86.53)$ & $59(86.76)$ & \\
\hline Previous intervention, $n(\%)$ & $42(80.7)$ & $56(82.35)$ & 0.86 \\
\hline Cause of PHG, $n(\%)$ & & & 0.37 \\
\hline Viral & $25(48.07)$ & $32(47.05)$ & \\
\hline Bilharzial & $5(9.61)$ & $7(10.29)$ & \\
\hline Mixed & $22(42.30)$ & $29(42.64)$ & \\
\hline Child classification, $n(\%)$ & & & 0.76 \\
\hline Child A & $8(15.38)$ & $11(16.17)$ & \\
\hline Child B & $17(32.69)$ & $22(32.35)$ & \\
\hline Child C & $27(51.92)$ & $35(51.47)$ & \\
\hline
\end{tabular}

No significant differences between the 2 groups regarding the baseline characteristics.

APC, argon plasma coagulation; ALT, alanine aminotransferase; AST, aspartate aminotransferase; INR, international normalized ratio; Hb, hemoglobin; WBC, white blood cells; TIBC, total iron-binding capacity; PHG, portal hypertensive gastropathy.

Our results revealed that there were 27 (22.5\%) patients with no esophageal varices; 20 patients of them were eradicated previously; and 7 had no esophageal varices. Regarding the severity of PHG according to NIEC, Bus accounts to about 58 (48.3\%), CRSs 53 (44.1\%), and Rolls 9 (7.5\%; Table 2). Furthermore, the fundus is the most affected site of the stomach 44 (36.6\%) followed by corporeal 41 (34.1\%) and finally pan gastric 35 (29.1\%). With regard to hepatic functional reserve, 19 (15.8\%) had Child class A, 39 (32.5\%) had Child class B, and 62 (51.6\%) had Child class C; this indicated that most patients with PHG had decompensated hepatic disease. In APC group, there was significant increase of $\mathrm{Hb}$, serum iron, serum ferritin and significant decrease of total iron-binding capacity (TIBC), at all postoperative check points as compared to pretreatment values in both per-protocol and intention-to-treat analysis $(p<$ 0.001; Table 3; Fig. 1). Additionally, there was gradual increase in the mean of $\mathrm{Hb}$, serum iron, and serum ferritin and gradual decrease of TIBC in Carvedilol group. Accordingly, there was overall improvement of IDA in both groups; however, it was significantly better in APC group than in Carvedilol group. Furthermore, there was reduction of transfusion requirements $>50 \%$ in transfusion-dependent patients over the following 3 months after the start of therapy in APC group in comparison to Carvedilol group. With regard to rebleeding in APC group, it was 
Fig. 1. Treatment outcomes after 3 months. $\mathrm{Hb}$, hemoglobin; TIBC, total iron-binding capacity; APC, argon plasma coagulation.

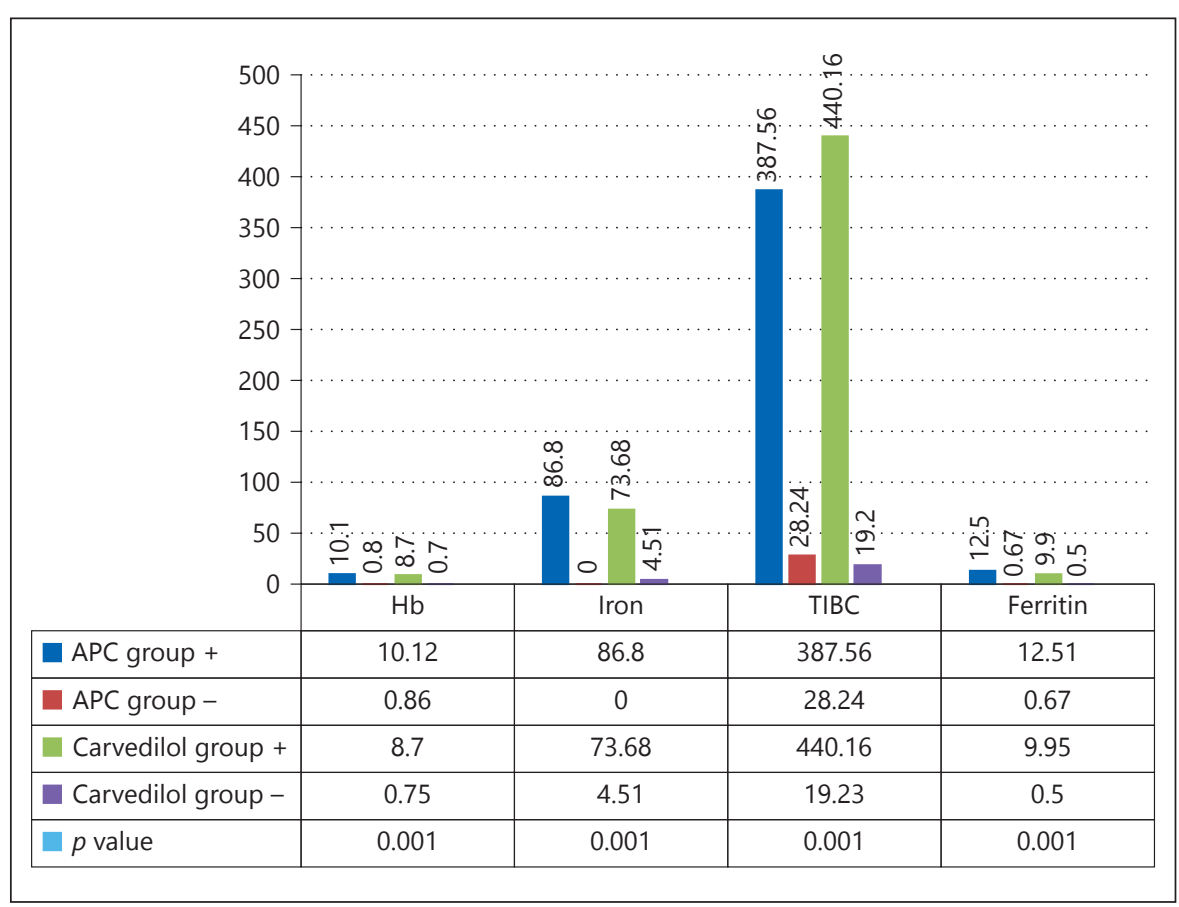

$28.84,17.30$, and $9.61 \%$, respectively, at 1,2 , and 3 months after treatment; while in Carvedilol group, it was 41.17, 34.61 , and $11.76 \%$, respectively, with significant difference between the 2 groups $p=0.02, p=0.03, p=0.05$, respectively, these results indicated that Carvedilol take long time to get a full benefit in preventing reloading in PHG. As regard to complications in APC group, gaseous distension was reported in $57.6 \%$ and pain at treatment site in $38.4 \%$. Concerning the APC sessions, the initial session duration extended from $22 \mathrm{~min}$ (fundic or corporeal) up to $30 \mathrm{~min}$ (pan gastric). The mean number of sessions was $1.65 \pm 0.8$, and the range of sessions was $1-4 ; 8$ (15.3\%) of patients needed 4 sessions, $12(23.07 \%)$ needed 3 sessions, 10 (19.2\%) patients needed 2 sessions, and 22 (42.30\%) needed only 1 session. The number of sessions varied according to PHG distribution; fundic and corporeal PHG needed 1 or 2 sessions, while pan gastric PHG needed 2, 3, or 4 sessions; therefore, patients with fundic and corporeal PHG required the lowest number of sessions, on the other hand, more sessions were needed for pan gastric PHG.

\section{Discussion}

This study investigated the efficacy and safety of APC versus Carvedilol in the treatment of patients with severe PHG. PHG is one of the clinical conditions that can cause
Table 2. Endoscopic evaluations of the study groups

\begin{tabular}{lclc}
\hline Variable & $\begin{array}{l}\text { APC group } \\
(n=52)\end{array}$ & $\begin{array}{l}\text { Carvedilol } \\
\text { group }(n=68)\end{array}$ & $p$ value \\
\hline PHG distribution, $n(\%)$ & & & 0.56 \\
$\quad$ Fundic & $19(36.53)$ & $25(36.76)$ & \\
$\quad$ Corporeal & $18(34.61)$ & $23(33.82)$ & \\
$\quad$ Pangastric & $15(28.84$ & $20(29.41)$ & \\
Severity of PHG, $n(\%)$ & & & 0.69 \\
$\quad$ MLP & $0(0)$ & $0(0)$ & \\
RPLS & $4(7.69)$ & $5(7.35)$ & \\
CRSs & $23(44.23)$ & $30(42.64)$ & \\
$\quad$ BBSs & $25(48.07)$ & $33(48.52)$ & \\
OV grade, $n(\%)$ & & & 0.88 \\
$\quad$ Eradicated & $12(23.07)$ & $15(22.05)$ & \\
$\quad$ Grade I & $14(26.92)$ & $18(26.47)$ & \\
$\quad$ Grade II & $20(38.46)$ & $26(38.23)$ & \\
$\quad$ Grade III & $6(11.53)$ & $9(13.23)$ & \\
Fundal varecis, $n(\%)$ & $8(15.38)$ & $10(14.70)$ & 0.30 \\
\hline
\end{tabular}

The fundus is the most affected site of the stomach 44 (36.6\%) followed by corporeal 41 (34.1\%) and finally pangastric 35 (29.1\%). APC, argon plasma coagulation; PHG, portal hypertensive gastropathy; MLP, mosaic-like pattern; RPLs, red-point lesions; CRSs, cherry-red spots; BBSs, black-brown spotsare. 
Table 3. Treatment outcomes

\begin{tabular}{llccc}
\hline Posttreatment & Test & $\begin{array}{c}\text { APC group } \\
(n=52)\end{array}$ & $\begin{array}{c}\text { Carvedilol group } \\
(n=68)\end{array}$ & $p$ value \\
\hline \multirow{2}{*}{ month } & Hb & $8.60 \pm 0.72$ & $7.75 \pm 0.49$ & $<0.001$ \\
& Iron & $68.81 \pm 4.54$ & $56.10 \pm 3.95$ & $<0.001$ \\
& TIBC & $450.13 \pm 22.77$ & $480.50 \pm 19.88$ & $<0.001$ \\
& Ferritin & $11.52 \pm 0.55$ & $9.24 \pm 0.66$ & $<0.001$ \\
& Rebleading & $15(28.84)$ & $28(41.17)$ & 0.02 \\
\hline \multirow{2}{*}{ months } & Hb & $9.51 \pm 0.60$ & $8.23 \pm 55$ & $<0.001$ \\
& Iron & $78.75 \pm 4.12$ & $67.95 \pm 4.19$ & $<0.001$ \\
& TIBC & $409.15 \pm 27.87$ & $465.26 \pm 19.05$ & $<0.001$ \\
& Ferritin & $11.95 \pm 0.85$ & $9.51 \pm 0.4$ & $<0.001$ \\
& Rebleading & $9(17.30)$ & $18(34.61)$ & 0.03 \\
\hline
\end{tabular}

Values are expressed as mean \pm SD or $n(\%)$.

Throughout follow-up period, There was significant increase in hemoglobin level, serum iron, and serum ferritin with a significant decrease in TIBC in APC group as compared to Carvedilol group.

There was gradual increase in the mean of hemoglobin, serum iron, and serum ferritin and gradual decrease of TIBC in Carvedilol group.

APC, argon plasma coagulation; Hb, hemoglobin; TIBC, total iron-binding capacity.

chronic GI hemorrhage in patients with cirrhosis and is manifested by chronic anemia [15]. A reduction in portal pressure is the main target of treatment in PHG. Nonselective beta-blockers are the most thoroughly investigated drugs for a sustained reduction in portal pressure. They should be continued on a long-term basis because discontinuing the drug frequently leads to recurrence of bleeding [16]. APC is an effective and safe method to stop bleeding in patients with PHG [7]. Our results revealed that most common cause of liver disease was HCV infection either alone or associated with bilharziasis. Our results do agree with the Egyptian Demographic Health Survey, who conducted survey including HCV biomarkers in 2008 on a large nationally representative sample and estimated that HCV prevalence among the 15-59 years age group to be $14.7 \%$ [17]. Therefore, Egypt has the highest HCV prevalence in the world [18]. In the current study, the number of patients with Child $\mathrm{B}$ and $\mathrm{C}$ scores was $84.16 \%$, which was higher than the number of patients with Child A score (15.83\%), and this finding was similar to Bang et al. [19] who reported that PHG is more frequently observed in patients with more severe liver disease. Our results demonstrated that PHG was higher in the fundus and body than the antrum. These results were found to be close to those reported by Bang et al. [19] who stated that PHG lesions are most often typically found in the fundus and body (proximal to antrum). Our results revealed also that $20 \%$ of cases had history of variceal intervention (sclerotherapy or band ligation) and this was similar to Elwakil et al. [20] who reported that variceal obliteration aggravates PHG. Furthermore, El-Khayat et al. [21] documented that endoscopic therapy has been associated with an increased incidence of PHG. On the other hand, another study observed no difference in the natural history of PHG whether or not previous endoscopic sclerotherapy therapy was performed [22]. APC is a new, efficacious, safe, and easy-to-use method for the noncontact application of high-frequency current through ionized and electrically conductive argon gas. It has been used successfully to treat vascular bleeding of the upper digestive tract including GAVE, sporadic angiodysplasia, hemorrhagic telangiectasia, and radiation-induced enteropathy [23]. We applied APC to all areas of visible red spots of congested gastric mucosa, and the end point of successful endoscopic therapy was production of a white coagulum. On comparing laboratory investigations in 1st visit then 1,2 , and 3 months of APC group, there was a highly sig- 
nificant increase in $\mathrm{Hb}$ level, serum iron, and serum ferritin with a significant decrease in TIBC as compared to Carvedilol group $(p<0.001)$ due to discontinuing of chronic blood loss from PHG. Additionally, these results may be attributed to coagulation of mucosal vessels, thus prevention of bleeding and improvement in gastric perfusion due to decrease in gastric congestion and therefore enhances absorption of the nutrients through gastric mucosa. Therefore, APC proved to be efficacious in controlling bleeding from PHG with no complications and with a significant improvement in Hb levels and a decrease in blood transfusions requirements. Another study evaluated the role of APC in treating different types of gastric vascular ectasia lesions in patients admitted for upper GI tract hemorrhage and revealed that the overall success of APC was $86 \%$, with only one recurrence of upper GI tract bleeding during the follow-up period, The total number of APC sessions was $1.9 \pm 1.3$, and the treatment success for PHG was $81 \%$ with a rise in hematocrit from baseline values $(p=0.01)$ [24]. Furthermore, there was gradual increase in the mean of $\mathrm{Hb}$, serum iron, and serum ferritin and gradual decrease of TIBC in Carvedilol group. Accordingly, there was overall improvement of IDA in both groups; however, it was significantly better in APC group than in Carvedilol group. Therefore, the combination of APC and Carvedilol, unless contraindicated, may have a synergistic effect in controlling PHG. The effect of nonselective beta-blockers may take longer time so in case of acute bleeding APC is a rapid, effective method for the control of PHG-induced bleeding, especially when beta-blockers are hazardous or contraindicated. Our results were found to be close to Abd El-Ghany et al. [25] who reported that the mean $\mathrm{Hb}$ pre-APC was $8.3 \pm 1.1$, which gradually elevated to become $10.72 \pm$ 1.54, this elevation showed significance throughout treatment follow-up. Moreover, our results do agree with that reported by Gonzalez-Suarez et al. [7] that studied the effect of APC on bleeding from PHG, where out of 22 cirrhotic patients, 16 patients had PHG with chronic anemia and 6 patients had PHG with acute bleeding episode. APC was applied to the area of PHG. Patients were followed up for a mean of 36 months. Hb value was significantly improved after APC. Another study has challenged the traditional consideration that lesions associated to PHG do not benefit from endoscopic therapy by evaluating the use of APC in the treatment of PHG [24] in this study, 11 patients with bleeding from PHG were included. APC sessions were aimed to ablate the greatest area of mucosal surface as possible, at least $80 \%$ in diffuse lesions. Sessions were repeated every 2-4 weeks. The end point, which was defined by the absence of upper GI bleeding or a reduction in transfusion requirements, was achieved in $81 \%$ of the patients who were bleeding from PHG. In the current study, there were no reported APC complications among the studied groups of patients apart from mild gaseous distension and local pain at epigastrium although these effects are temporary and mild. This do agree with Abd El-Ghany et al. [25] who reported that there were no complications related to APC throughout 4 months' treatment follow-up. After performing the APC and follow-up for 1 year, APC proved to be efficacious in controlling bleeding from PHG with no complications and with a significant improvement in $\mathrm{Hb}$ levels and a decrease in blood transfusions and ICU admissions $(p<0.0001)$. On the other hand, there has been several studies on the use of Carvedilol for $\mathrm{PH}$ in patients with cirrhosis. Most of these studies assessed the effects of Carvedilol on HVPG. The results show that Carvedilol causes dose-related and marked decreases in HVPG (of about 20\% from baseline) significantly greater than those caused by propranolol [26]. Banares et al. [27] reported the longest follow-up trial of $11.1 \pm 4.1$ weeks in 51 cirrhotic patients (26/carvidelol, 25/propranolol). The Carvedilol doses were administrated at $12.5-50 \mathrm{mg}$ (mean $31 \pm 4 \mathrm{mg} /$ day) starting at $6.25 \mathrm{mg}$ and titrated up every 4 days according to blood pressure and heart rate. Chronic Carvedilol administration resulted in 58\% hemodynamic response rate compared to $23 \%$ response rate in the propranolol group. Chronic Carvedilol administration at lower doses of $12.5 \mathrm{mg}$ was studied in 2 clinical trials $[12,28]$. Both trials reported a reduction in HVPG by $23-43 \%$ from baseline measurements without a significant effect on the mean arterial blood pressure. Regarding our limitations in the current study, we included an adequate number of patients; however, it should be confirmed with a larger sample size. Additionally, the follow-up period of our study was short and it was better to extend to sufficient follow-up period. In conclusion, after 3 months follow-up in managing PHG, we have found that APC significantly improve IDA and decrease transfusion requirements in patients with severe PHG as compared to oral Carvedilol with small risk of adverse events. Additionally, the combination of APC and Carvedilol, unless contraindicated, may have a synergistic effect in controlling PHG.

\section{Disclosure Statement}

The authors declared that they have no conflict of interest. 


\section{References}

1 Bosch J, Berzigotti A, Garcia-Pagan JC, Abraldes JG. The management of portal hypertension: rational basis, available treatments and future options. J Hepatol. 2008;48(suppl 1): S68-92.

2 Primignani M, Carpinelli L, Preatoni P, Battaglia G, Carta A, Prada A, et al. Natural history of portal hypertensive gastropathy in patients with liver cirrhosis. The New Italian Endoscopic Club for the study and treatment of esophageal varices (NIEC). Gastroenterology. 2000 Jul;119(1):181-7.

3 Garcia-Tsao G. Current management of the complications of cirrhosis and portal hypertension: variceal hemorrhage, ascites, and spontaneous bacterial peritonitis. Gastroenterology. 2001 Feb;120(3):726-48.

4 McCormack TT, Sims J, Eyre-Brook I, Kennedy H, Goepel J, Johnson AG, et al. Gastric lesions in portal hypertension: inflammatory gastritis or congestive gastropathy? Gut. 1985 Nov;26(11):1226-32.

5 Spina GP, Arcidiacono R, Bosch J, Pagliaro L, Burroughs AK, Santambrogio R, et al. Gastric endoscopic features in portal hypertension: final report of a consensus conference, Milan, Italy, September 19, 1992. J Hepatol. 1994 Sep; 21(3):461-7.

6 Sato T, Yamazaki K, Toyota J, Karino Y, Ohmura T, Akaike J, et al. Efficacy of argon plasma coagulation for gastric antral vascular ectasia associated with chronic liver disease. Hepatol Res. 2005 Jun;32(2):121-6.

7 Gonzalez-Suarez D, Monfort D, Piqueras M, Planella M, Aracil C, Gallego A, et al. Endoscopic treatment with Argon plasma coagulation for portal hypertensive gastropathy with no response to pharmacological therapy [abstract]. J Hepatol. 2003;38:59.

8 Boyer TD. Pharmacologic treatment of portal hypertension: past, present, and future. Hepatology. 2001 Oct;34(4 Pt 1):834-9.

9 Hemstreet BA. Evaluation of carvedilol for the treatment of portal hypertension. Pharmacotherapy. 2004 Jan;24(1):94-104.

10 Frishman WH. Carvedilol. N Engl J Med. 1998 Dec;339(24):1759-65.

11 Hemstreet BA. Evaluation of carvedilol for the treatment of portal hyperten- sion. Pharmacotherapy. 2004 Jan;24(1): 94-104.

12 Tripathi D, Therapondos G, Lui HF, Stanley AJ, Hayes PC. Haemodynamic effects of acute and chronic administration of low-dose carvedilol, a vasodilating beta-blocker, in patients with cirrhosis and portal hypertension. Aliment Pharmacol Ther. 2002 Mar;16(3): 373-80.

13 Westaby D, Macdougall BR, Melia W, Theodossi A, Williams R. A prospective randomized study of two sclerotherapy techniques for esophageal varices. Hepatology. 1983 SepOct;3(5):681-4.

14 Tripathi D, Stanley AJ, Hayes PC, Patch D, Millson C, Mehrzad H, et al. Clinical Services and Standards Committee of the British Society of Gastroenterology. U.K. guidelines on the management of variceal haemorrhage in cirrhotic patients. Gut. 2015;64:1680-704.

15 Mathurin SA, Agüero AP, Dascani NA, Prestera JA, Gianserra C, Londero E, et al. [Anemia in hospitalized patients with cirrhosis: prevalence, clinical relevance and predictive factors]. Acta Gastroenterol Latinoam. 2009; 39:103-11.

16 Sarin SK, Agarwal SR. Gastric varices and portal hypertensive gastropathy. Clin Liver Dis. 2001 Aug;5(3):727-67.

17 El-Zanaty F, Way A (2008) MACRO International. Egypt demographic and Heath Survey, 2008. Final report. In: Measure DHS.

18 Sievert W, Altraif I, Razavi HA, Abdo A, Ahmed EA, Alomair A, et al. A systematic review of hepatitis $C$ virus epidemiology in Asia, Australia and Egypt. Liver Int. 2011 Jul; 31(suppl 2):61-80.

19 Bang CS, Kim HS, Suk KT, Kim SE, Park JW, Park SH, et al. Portal hypertensive gastropathy as a prognostic index in patients with liver cirrhosis. BMC Gastroenterol. 2016 Aug; 16(1):93.

20 Elwakil R, Al Breedy AM, Gabal HH. Effect of endoscopic variceal obliteration by band ligation on portal hypertensive gastro-duodenopathy: endoscopic and pathological study. Hepatol Int. 2016 Nov;10(6):965-73.

21 El-Khayat HR, El Khattib A, Nosseir M, Ezz MA, El-Amin H, Fouad YM. Portal hyper- tensive enteropathy before and after variceal obliteration: an endoscopic, histopathologic and immunohistochemical study. J Gastrointestin Liver Dis. 2010 Jun;19(2): 175-9.

22 Gjeorgjievski M, Cappell MS. Portal hypertensive gastropathy: A systematic review of the pathophysiology, clinical presentation, natural history and therapy. World J Hepatol. 2016 Feb;8(4):231-62.

23 Roman S, Saurin JC, Dumortier J, Perreira A, Bernard G, Ponchon T. Tolerance and efficacy of argon plasma coagulation for controlling bleeding in patients with typical and atypical manifestations of watermelon stomach. Endoscopy. 2003 Dec;35(12):1024-8.

24 Herrera S, Bordas JM, Llach J, Ginès A, Pellisé M, Fernández-Esparrach G, et al. The beneficial effects of argon plasma coagulation in the management of different types of gastric vascular ectasia lesions in patients admitted for GI hemorrhage. Gastrointest Endosc. 2008 Sep;68(3):440-6.

25 Abd El-Ghany S, El-taher S, El-Zefzafy W, Sabal A. Safety and efficacy of endoscopic treatment with argon plasma coagulation of gastric bleeding in cases of gastric antral vascular ectasia and portal hypertensive gastropathy. AAMJ. 2014;12(1)

26 Fontana RJ, Sanyal AJ, Mehta S, Doherty MC, Neuschwander-Tetri BA, Everson GT, et al.; HALT-C Trial Group. Portal hypertensive gastropathy in chronic hepatitis $\mathrm{C}$ patients with bridging fibrosis and compensated cirrhosis: results from the HALT-C trial. Am J Gastroenterol. 2006 May;101(5):98392.

27 Banares R, Moitinho E, Piqueras B, Casado M, Garcia-Pagan JC, De Diego A, et al. Carvedilol, a new nonselective beta-blocker with intrinsic anti- Alpha1-adrenergic activity, has a greater portal hypotensive effect than propranolol in patients with cirrhosis. Hepatology. 1999 Jul;30(1):79-83.

28 De BK, Das D, Sen S, Biswas PK, Mandal SK, Majumdar D, et al. Acute and 7-day portal pressure response to carvedilol and propranolol in cirrhotics. J Gastroenterol Hepatol. 2002 Feb;17(2):183-9. 